\title{
Development of a Textile Based Protein Sensor for Monitoring the Healing Progress of a Wound
}

\author{
Yomna ElSaboni ( $\nabla$ yomna.elsaboni@ntu.ac.uk) \\ Nottingham Trent University \\ John A. Hunt \\ Medical Technologies Innovation Facility, Nottingham Trent University \\ Christine Moffatt \\ Nottingham University Hospital \\ Yang Wei \\ Nottingham Trent University
}

\section{Research Article}

Keywords: Protein Sensor, Wound, polyester nonwoven fabric

Posted Date: January 7th, 2022

DOI: https://doi.org/10.21203/rs.3.rs-1228058/v1

License: (9) This work is licensed under a Creative Commons Attribution 4.0 International License.

Read Full License 


\title{
Development of a textile based protein sensor for monitoring the healing progress of a wound
}

\author{
Yomna ElSaboni ${ }^{1, *}$, John A. Hunt ${ }^{2,3}$, Christine Moffatt ${ }^{4}$, and Yang Wei ${ }^{1}$ \\ ${ }^{1}$ Smart wearable research group, Department of Engineering, School of Science and Technology, Nottingham Trent \\ University, NG11 8NS Nottingham, UK \\ ${ }^{2}$ Medical Technologies Innovation Facility, Nottingham Trent University, Nottingham, NG11 8NS, U.K \\ ${ }^{3}$ College of Biomedical Engineering, China Medical University, Taichung 40402, Taiwan \\ ${ }^{4}$ Skin Integrity, Institute care excellence, Nottingham University Hospital, Nottingham, NG5 1PB, UK \\ *yomna.elsaboni@ntu.ac.uk
}

\begin{abstract}
This article focuses on the design and fabrication of flexible textile-based protein sensors to be embedded in wound dressings. Chronic wounds require continuous monitoring to prevent further complications and to determine the best course of treatment in the case of infection. As proteins are essential for the progression of wound healing, they can be used as an indicator of wound status. Through measuring protein concentrations, the sensor can assess and monitor the wound condition continuously as a function of time. The protein sensor consists of electrodes that are directly screen printed using both silver and carbon composite inks on polyester nonwoven fabric which was deliberately selected as this is one of the common backing fabrics currently used in wound dressings. Three sensor designs were investigated to determine if any were suitable for protein detection. These sensors were experimentally evaluated and compared to each other by using albumin protein in phosphate buffered saline (PBS). A comprehensive set of cyclic voltammetry measurements were used to determine the optimal sensor design to provide the measurement of protein in solution. The best sensor was comprised of only silver conductive ink present to form the tracks outside the interface zone and a carbon only layer in the working and counter electrodes at the interface zone. This design prevents the formation of silver dioxide and protects the sensor from rapid decay, which allows for the recording of consecutive measurements using the same sensor. The chosen printed protein sensor was able to detect BSA at varying concentrations ranging from $30-0.3 \mathrm{mg} / \mathrm{ml}$ with a sensitivity of $0.0026 \mu \mathrm{A} / \mathrm{M}$.
\end{abstract}

\section{Introduction}

Skin is a crucial organ of the human body as it acts as a barrier to protect the rest of the body's tissues and organs ${ }^{1}$, therefore when it suffers an injury, other essential and healthy organs could become infected or injured ${ }^{2}$. While in most minor wound cases, minimal intervention is required such as placing a bandage or medical gauze to prevent damage, keep it clean and to prevent it being overrun by infectious microorganisms, whereas many chronic wounds need to be monitored and retreated constantly over long periods of time. The cost of treating wounds is always a critical issue as it is estimated to account for at least $3 \%$ of the total healthcare expenditure in most developed countries ${ }^{3}$. Additionally, since 2018 , it is estimated that the UK is managing approximately 3.8 million patients with a wound in a clinical setting annually ${ }^{4}$ It was estimated that health services in 2012 spent $£ 5.1$ billion on costs associated with wound care management ${ }^{5}$ which provides a compelling case for improvement in the current standard of wound dressings not only to reduce healthcare costs but also to improve patient quality of life ${ }^{6}$. However, better and effective means of reporting quantitative information about the wound condition in real time is required to inform and guide treatment decisions as improved wound care will deliver improved public health and healthcare $\operatorname{costs}^{7,8}$.

The wound healing process can be monitored by repeatedly determining the multiple physiological changes that occur including but not limited to $\mathrm{pH}$, alkalization, temperature, uric acid and specific protein types such as albumin and fibrinogen whilst tissue repair progresses ${ }^{2}$. Detection of these biomarkers with minimally invasive techniques can provide an effective way for the real-time monitoring of the condition of a wound. In addition, remote wound monitoring could keep the patient informed about their condition, improve their quality of life and reduce the frequency of face-to-face consultations and treatments with healthcare providers ${ }^{9}$.

Integrating electronics with textiles has advanced medical care by facilitating physiological parameters to be monitored remotely through minimal or noninvasive techniques and sometimes with reduced direct contact with the human body ${ }^{10}$. Improved flexibility and durability allows electronic devices to be more suitable for wearable biosensors as they can be embedded into clothing to realize electronic textiles ${ }^{11}$. These emerging technologies in wearable electronics have made using 
smart textiles in the design of flexible wound dressings more achievable. The aim of wearable textile biosensors is to indirectly detect critical physiological changes in the body through measuring indirect stimuli that can be readily detected from outside the body despite the uncontrolled environment surrounding the body ${ }^{12}$. However, to be embedded in wound dressings, not only does it have to be miniaturized but it also needs to be flexible and lightweight to make positioning around the wound feasible and as comfortable to wear as possible regardless of the location of the wound ${ }^{13}$. To provide better flexibility the ink used in fabricating the biosensor plays a critical role as adding a bendable layer to a textile can provide fabric reinforcement and make it mechanically stronger ${ }^{14}$.

The design of the protein sensor is based on the structure of an electrochemical cell that uses a three-electrode configuration to perform the cyclic voltammetry measurements ${ }^{15-17}$. Electrochemical sensors are generally preferred because they can provide rapid real time monitoring of change and wound conditions, they are also relatively inexpensive and can be miniaturized and embedded within textiles ${ }^{18}$. Categorically the device should be considered to be a potentiometric biosensor which works by measuring the voltage produced when electric current flows through the solution under static conditions ${ }^{19}$. Potentiometric analytical systems have been used in the past to detect a wide range of biomarkers including but not limited to penicillin, urea, glucose, carcinoembryonic antigen, cancer antigen, prostate-specific antigen and lysin, in addition to determination of $\mathrm{pH}$ levels ${ }^{19-24}$. These sensors have high selectivity, fast response times, ease of operation, wide dynamic range and are generally inexpensive to fabricate ${ }^{25,26}$ and most importantly in this application they can be converted into portable kits ${ }^{27}$, which is crucial when used in wound dressings.

To fabricate electrochemical sensors, a variety of manufacturing techniques have been implemented in the past such as photolithography ${ }^{28,29}$, roll-to-roll gravure printing ${ }^{30}$, e-beam evaporation ${ }^{31}$, dip coating and drop casting ${ }^{32}$, screen printing ${ }^{33}$. Screen printing technology has been used to fabricate electrochemical electrodes on ceramic or plastic based substrates ${ }^{34-36}$.This technique involves forcing suitable ink formulations in paste form through a patterned stencil or screened mesh of a specific size and shaped using a squeegee to form the desired design on a substrate ${ }^{37}$.

While $\mathrm{pH}$ and temperature have been used as parameters in assessing wound status ${ }^{38,39}$, detecting protein concentrations helps to identify wound healing stages ${ }^{40}$ as it is less likely to be affected by the active external environment surrounding the exudate. Albumin was the protein determined in this research work, which has been modelled and measured previously ${ }^{41,42}$ as it is the most abundant protein in blood plasma (it represents $50 \%$ of total protein) ${ }^{43}$, previous research has shown a relation between the wellness of a person and the albumin concentration ${ }^{44}$, establishing albumin concentration as a good marker of protein concentrations in wounds. Albumin concentrations in wounds have been used as indicative of wound severity conditions, for which a concentration of $>15 \mathrm{mg} / \mathrm{ml}$ is considered to be present in inflamed wounds ${ }^{45}$. While the protein level in a healing wound is around $9 \mathrm{mg} / \mathrm{ml}$, contrasted against levels of $35 \mathrm{mg} / \mathrm{ml}$ for chronic slow healing wounds ${ }^{46}$.

Bovine serum albumin (BSA) was used to prepare standard solutions of albumin ${ }^{47}$. The use of BSA as a protein source specifically when testing electro-chemical sensors has been previously reported by others ${ }^{48}$ because the concentration can be easily standardized and altered precisely to evaluate the detection range. Most importantly, the properties of BSA are very similar to human serum albumin with respect to other proteins as discussed in a recent paper ${ }^{42}$.

For the first time in literature, this research presents a unique approach to integrate protein sensors in fabric which improves the sensors durability, comfort, flexibility and wearability and performance within specification. Although a similar approach was presented recently ${ }^{7,49}$, where screen printed electrodes (SPEs) were printed on a paper and placed inside a bandage but measure $\mathrm{pH}$ and uric acid to monitor chronic wounds.

In this research, three designs were investigated to determine the optimal biosensor design for integration into wound dressings. These designs were fabricated by screen printing and were evaluated based on the electrical connectivity to make sure the tracks were not broken or forming a short circuit and then their electrochemical performance was determined to ensure sensitivity. Each design was printed directly on 3 different types of fabric of varying surface roughness and layer thickness. The conductive tracks were made from silver flake-based ink and carbon ink. UV curable dielectric ink was used to smooth out the textile surface and to provide an even surface for further printing. The same UV ink was used as the encapsulation. 2-point resistance measurement was conducted on printed layers and cyclic voltammetry measurements using BSA solutions were performed.

\section{Results and Discussion}

The research had two main stages. The first stage encompassed the design and fabrication of the textile based sensors and the second stage covered the testing of the sensors using a previously established empirical technique reported by the authors ${ }^{41}$.

\section{Fabrication of textile based-screen printed electrodes}

The process of screen printing on fabric involves four stages as illustrated in Figure 1 which shows an exploded view of the three designs. The first design consisted of purely silver layer as both the conductive tracks and electrodes, the second consisted 
of a mix of both silver and carbon layers, while the third design had purely a carbon layer as part of the electrodes in the region where the sensor encounters the fluid under test at the interface zone. The dimensions of the sensors are shown in Figure2.

The sensors were printed onto three textiles, two of which are medical fabrics. The first one (Type A) was a polypropylene non-woven fabric and has areal surface roughness $(\mathrm{Sa}=119.24 \mu \mathrm{m})$, the second (Type $\mathrm{B}$ ) is a blend of cotton/polyester woven fabric and has areal surface roughness $(\mathrm{Sa}=59.37 \mu \mathrm{m})$ while the third one (Type $\mathrm{C}$ ) is made from polyester non-woven fabric areal surface roughness $(\mathrm{Sa}=151.52 \mu \mathrm{m})$. Each fabric was placed and adhered in turn onto an alumina tile which provided a rigid platform for printing. The sensors were successfully printed on all three types of fabrics but it was easier to print on the cotton/polyester fabric. However, using polyester non-woven fabric (Type C) which is similar to the one used in wound dressings prevented the initial substrate layer from cracking, while Type A fabric was more difficult to print on because it started to tear apart. Upon fabrication, the final printed sensors developed are shown in Figure 3. After the printed sensors were removed from the alumina, the resistance between two end points of each electrode of the printed tracks was measured using a multimeter. The resistance observed in the designs containing silver layer inside the interface area (design A and B shown in Figure 1) was less than $1 \mathrm{ohm}$ on average, while the carbon only design always had a much higher resistance (in the range of 260-410 ohms). Microscopic images of the three designs are shown in Figure 4. In all three designs, there were three electrodes (working, counter, reference electrodes) as illustrated in the Figure 4.(A) consisted of purely silver tracks throughout the design of the sensor, while the second design shown in (B) had a carbon layer on top of the silver layer on the working and counter electrode in the area where the sensor encounters the solution under test.Finally (C) shows a sensor that only had carbon ink deposited on the working and counter electrodes where the sensor encounters the solution under test which joins the silver metal tracks. As can be seen in the image the tracks were well defined and were separated from each other to prevent short circuit.

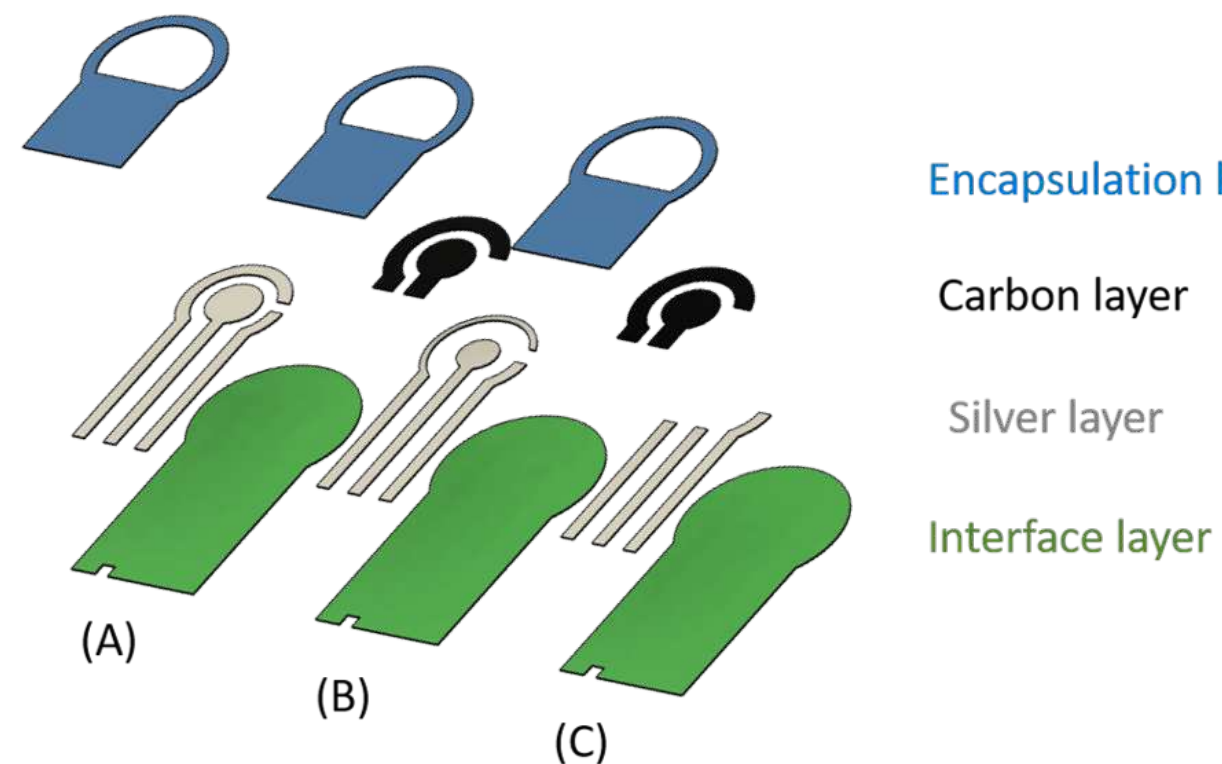

Figure 1. The three different SPE biosensors designs

The main challenge in the printing process was to maintain the correct alignment when printing each layer to prevent short circuit and to preserve the sensor design. To resolve this issue, a trial print was deposited on a transparent laminate sheet before every deposit to make sure the patterns are all perfectly aligned before printing directly on fabric. The second main challenge faced repeatedly was the roughness of each layer. To print several layers on top of each other, all layers need to be smooth with no pin holes on the surface as this affected the roughness of the final finishing layer. To address this issue, the smooth side of the fabric was initially shaved to enable the surface to be as uniform as possible. The dielectric layer was then printed several times with varying printing gaps to reduce any pin holes from forming on the deposited layer. The third challenge faced upon fabrication was that the fabric could not be easily removed from the alumina. It was observed that this only occurred when using thin fabric such as Type A fabric shown in Figure 3. To avoid this issue, two layers of the same fabric were adhered and fixed on top of each other. This strengthened the fabric layer and made it easier to remove after printing.

The printed layers after each stage are shown in Figure 5. Initially, in the first print shown in (A), the polymer interface layer was deposited six times to create a smooth platform for subsequent layers to be placed on top. The interface layer was then cured under UV light to produce a thickness of $110 \mu \mathrm{m}$. Next, the silver conductive electrodes were printed as shown in 

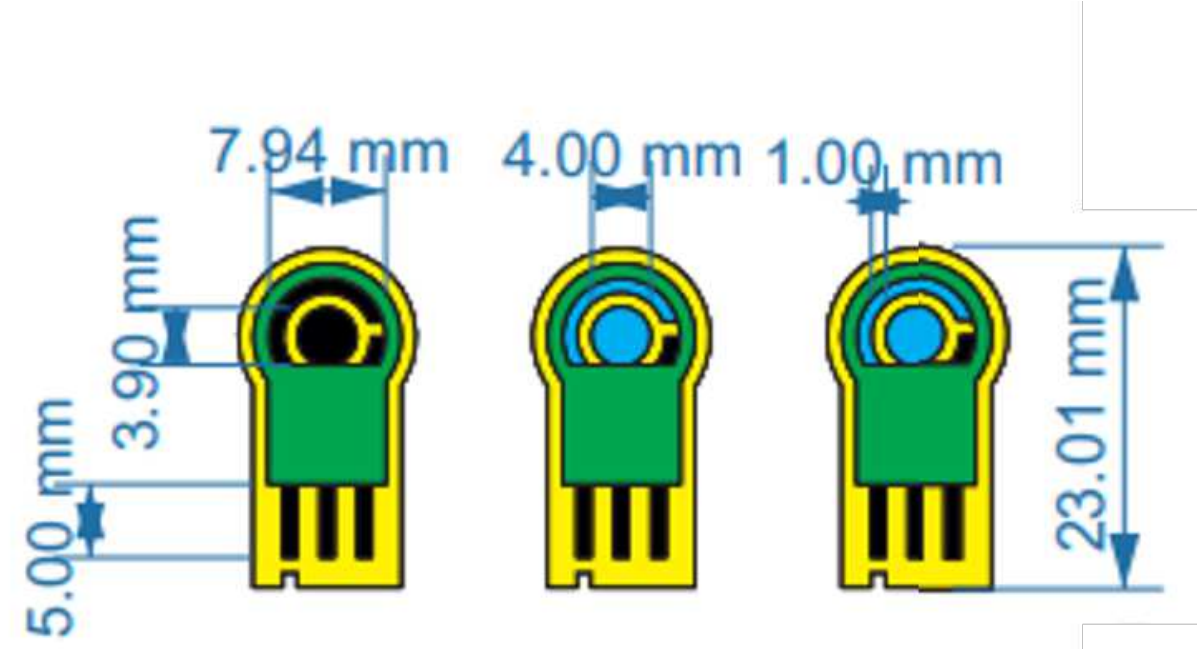

Figure 2. The dimensions of the three screen printed sensors types

(B) and placed in the oven for 15 minutes at $100^{\circ} \mathrm{C}$. The silver layer was printed twice and produced a thickness of $16 \mu \mathrm{m}$. The carbon layer was then printed only on the second and third designs as shown in Figure 5(C) and placed in the oven for 15 minutes under $100{ }^{\circ} \mathrm{C}$. The print was repeated twice to create a thickness of $26 \mu \mathrm{m}$. Finally, the same material used for the interface layer was printed to protect the conductive tracks of the sensors as shown in (D) and was cured under UV light to produce a thickness of $14 \mu \mathrm{m}$.

Upon fabrication, the sensors were highly flexibly and can be easily bent as shown in Figure 6, where the sensor was wrapped around a $1 \mathrm{~mL}$ pipette tip with $4 \mathrm{~mm}$ bending radius facing inwards on the left and outwards on the right. The flexibility presents a silver and carbon based screen printed electrodes (SPEs) bent to demonstrate its ability to wrap around an object without causing any cracks in any of the conductive electrodes or tracks and the interface layer. This feature is of great significance because of the nature of the targeted application as the exact positioning of a wound is unpredictable, and the dressing does need to be wrapped and bent around it.

\section{Cyclic voltammetry measurements}

After fabricating the sensors, the fabric around the bottom edges of the sensor was removed in order to be connected to the AUTOLAB Dropsens adaptor which includes an insertion connector with 3 pins. BSA with 8 concentrations ranging from 0.3 $30 \mathrm{mg} / \mathrm{ml}$ were used as the protein sources. Initially, the experiment was conducted on all three sensor designs on three types of fabrics to establish the most suitable design. For each measurement, the current at the oxidation peak was observed and recorded and a best fit line of all the measurements was plotted in Figure 7. The SPE design with only silver stopped working when testing a solution with concentration below $3 \mathrm{mg} / \mathrm{ml}$. As shown in Figure 7, the two cycles conducted using SPE with only carbon were the closest to each other in terms of gradient as there was less than $8 \%$ difference in comparison with design $\mathrm{A}$ and $\mathrm{B}$ where the differences in measurements between two cycles were $32 \%$ and $41 \%$, respectively. This demonstrated that design $\mathrm{C}$ was the most stable and reliable design as it provided reproducible and comparable results each time.

The status of the sensors after the cyclic voltammetry measurement was investigated to ensure no printed material was removed. This was done by examining each sensor post measurement under the microscope. This is important because the formation of a dark grey color (Silver dioxide) coating occurred after testing some sensor samples due to the oxidation reaction that took place at the working electrode and mostly visible in the silver only based SPE as shown in Figure 8 . This indicated that the silver electrode got damaged before the end of the cycle and could lead to unreliable results. In order to examine the underlying silver layer in design B, the carbon layer was manually removed to expose the silver layer. As shown in the microscopic image, SPE design (B) where the carbon layer was scratched to make the silver layer more visiblewas still oxidized, but it was far less visibly damaged than design (A) because it is protected by the carbon layer as it is noncorrosive. The SPE design $(C)$ remained unchanged as there was no silver present at the interface zone.

Further empirical testing was only conducted using design (C) SPEs. The cyclic voltammetry after the redox reaction is shown in Figure 9 when $30 \mathrm{mg} / \mathrm{ml} \mathrm{BSA}$ was used. The experiment was repeated three times on the same sensor and performed on all three types of fabric. The results obtained using the three fabrics overlapped each other and were within the same current range at the working electrode $(0.06-0.15 \mu \mathrm{A})$. Therefore, the effect of the type of fabric on the redox reaction was minimal. Type (C) fabric was more repeatable as clearly shown in the graph (the undashed lines) and was chosen as the standard in 


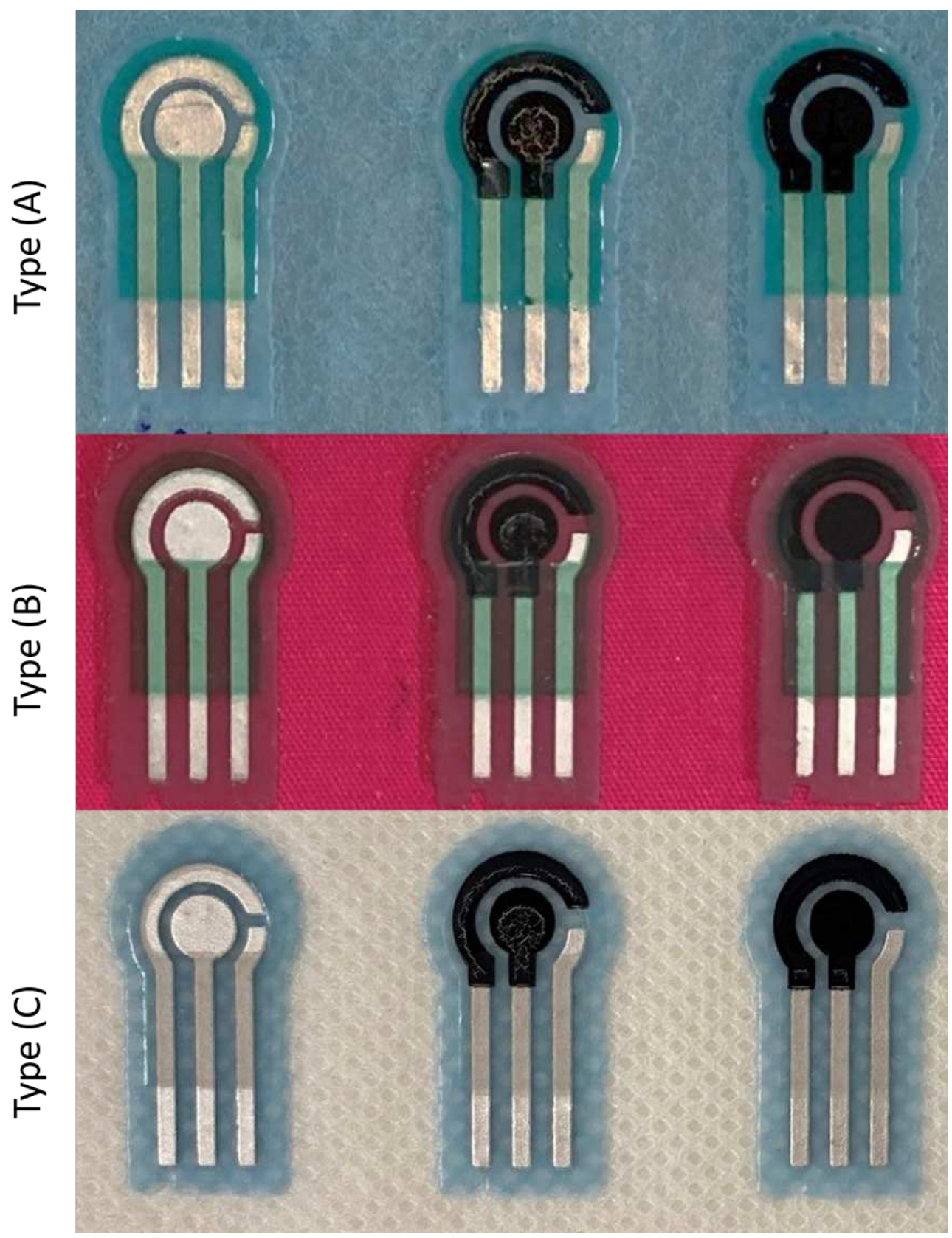

Figure 3. The design of the final three sensors screen printed on the three types of fabrics

the fabrication of the sensor as it is also commonly used for medical wound dressings. The first cycle was always lower in magnitude than the measurements obtained in the subsequent cycles. The second and third cycles were consistently closer to each other in comparison with the initial cycle. This is more visible when all peaks of several concentrations of each cycle were compared to each other as shown in Figure 10. Therefore, as a standard it is best to take the average of the second and third cycles when comparing the results of several BSA concentrations.

Since the combination of type (C) fabric and SPE design (C) was ideal, eight BSA concentrations were used to examine the sensitivity of this combination. The current observed at the oxidation peaks were recorded, shown in Figure 10. The best fit line was drawn based on the average value around each solution of each cycle. While the initial cycle was consistently lower in gradient as presented in Table 1 than the second and third cycles conducted on the same un-replaced sensor which illustrates that SPE design (C) is the most reproducible. 


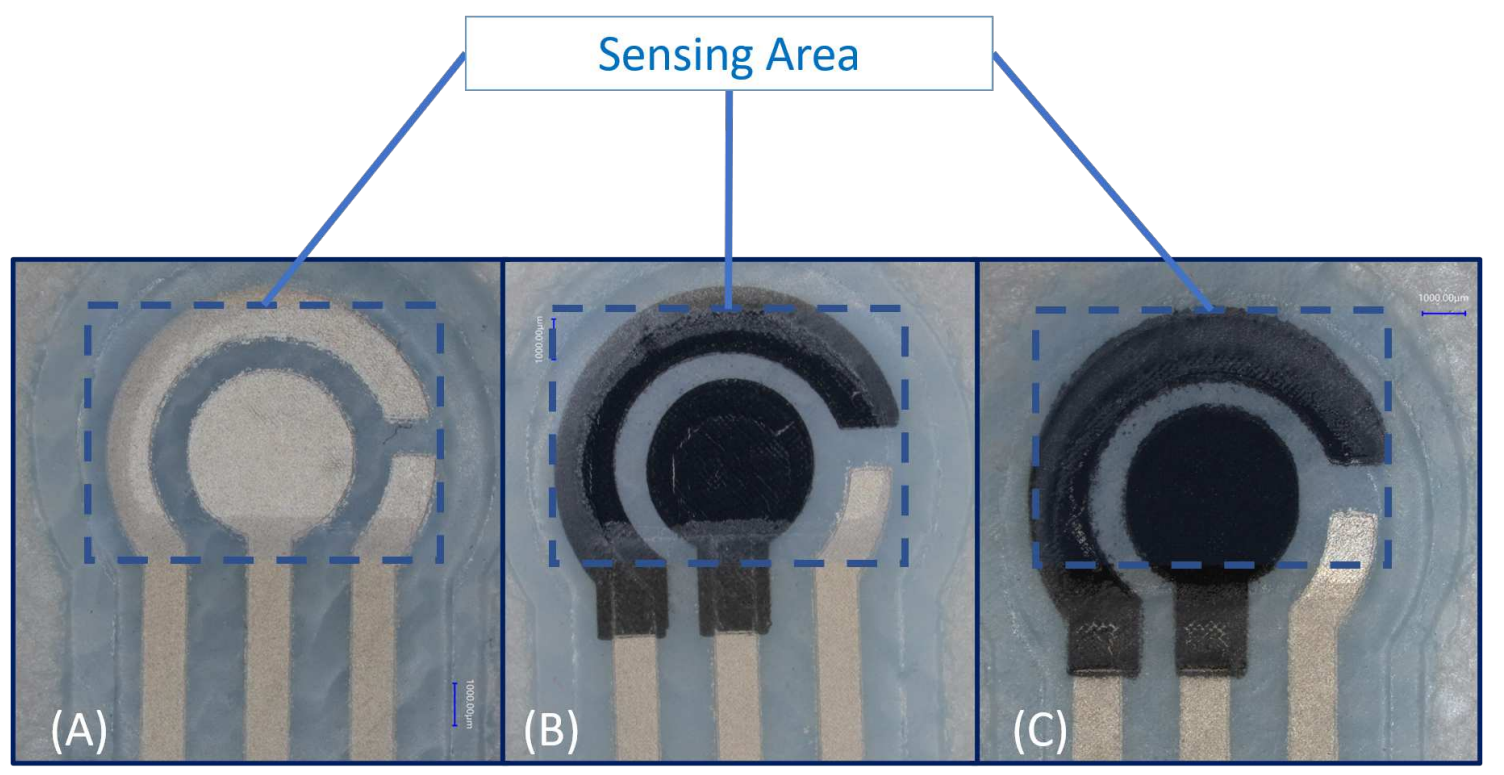

Figure 4. Microscopic images of the three textile printed sensors. (A) Silver only based screen printed sensors, (B) Carbon and Silver based screen printed sensors and (C) Carbon only based screen printed sensors

(A)

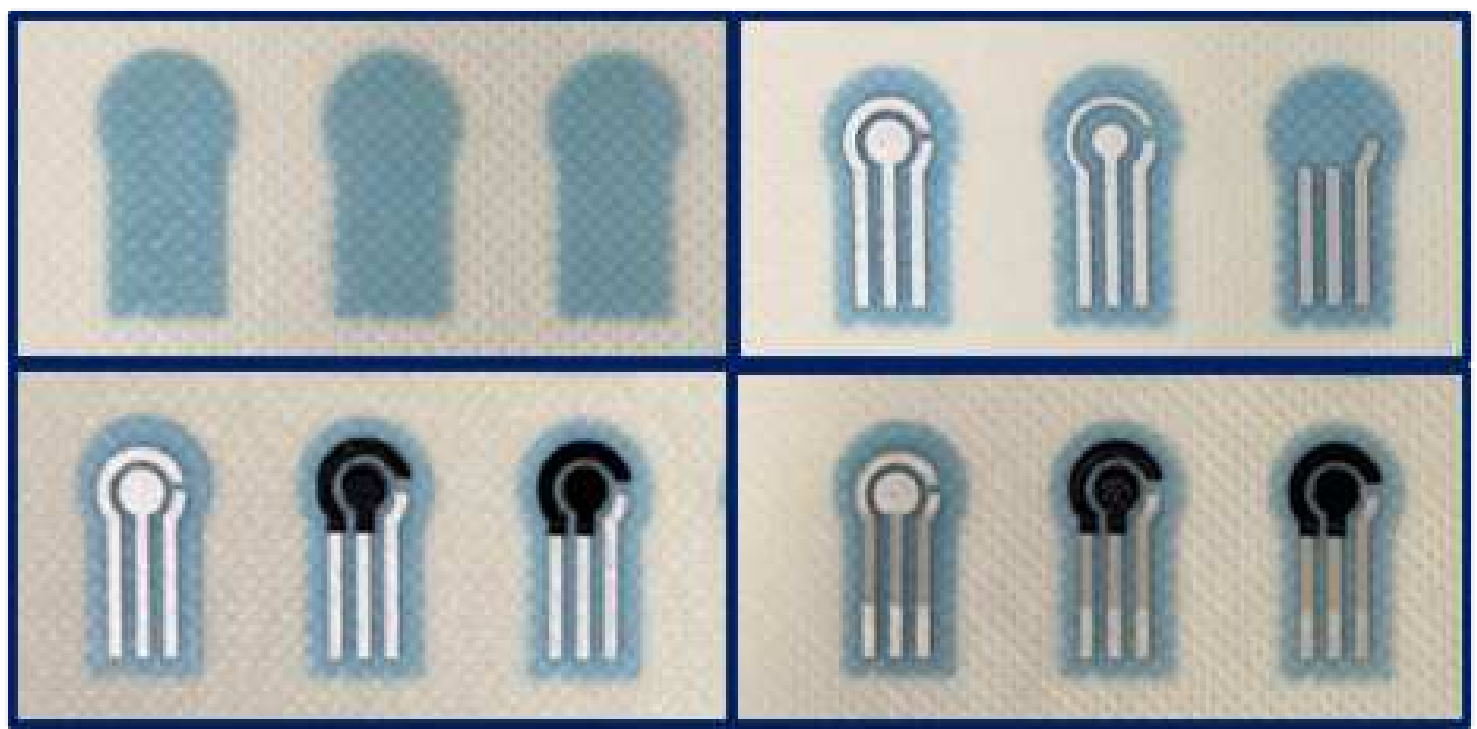

(B)

(D)

Figure 5. The different stages of screen printing the carbon electrodes (A) interface layer, (B) Silver, (C) Carbon, (D) Encapsulation

\begin{tabular}{|c|c|c|}
\hline SPE Type & Sensitivity $(\mu \mathbf{A} / \mathbf{M})$ & zero-point $(\mu \mathbf{A})$ \\
\hline Design (A) Trial 1 & 18 & 10 \\
\hline Design (A) Trial 2 & 13 & 120 \\
\hline Design (B) Trial 1 & 0.0034 & 0.04 \\
\hline Design (B) Trial 2 & 0.0052 & 0.038 \\
\hline Design (C) Trial 1 & 0.0024 & 0.012 \\
\hline Design (C) Trial 2 & 0.0026 & 0.009 \\
\hline
\end{tabular}

Table 1. The best fit line parameters of oxidation peak measurements 


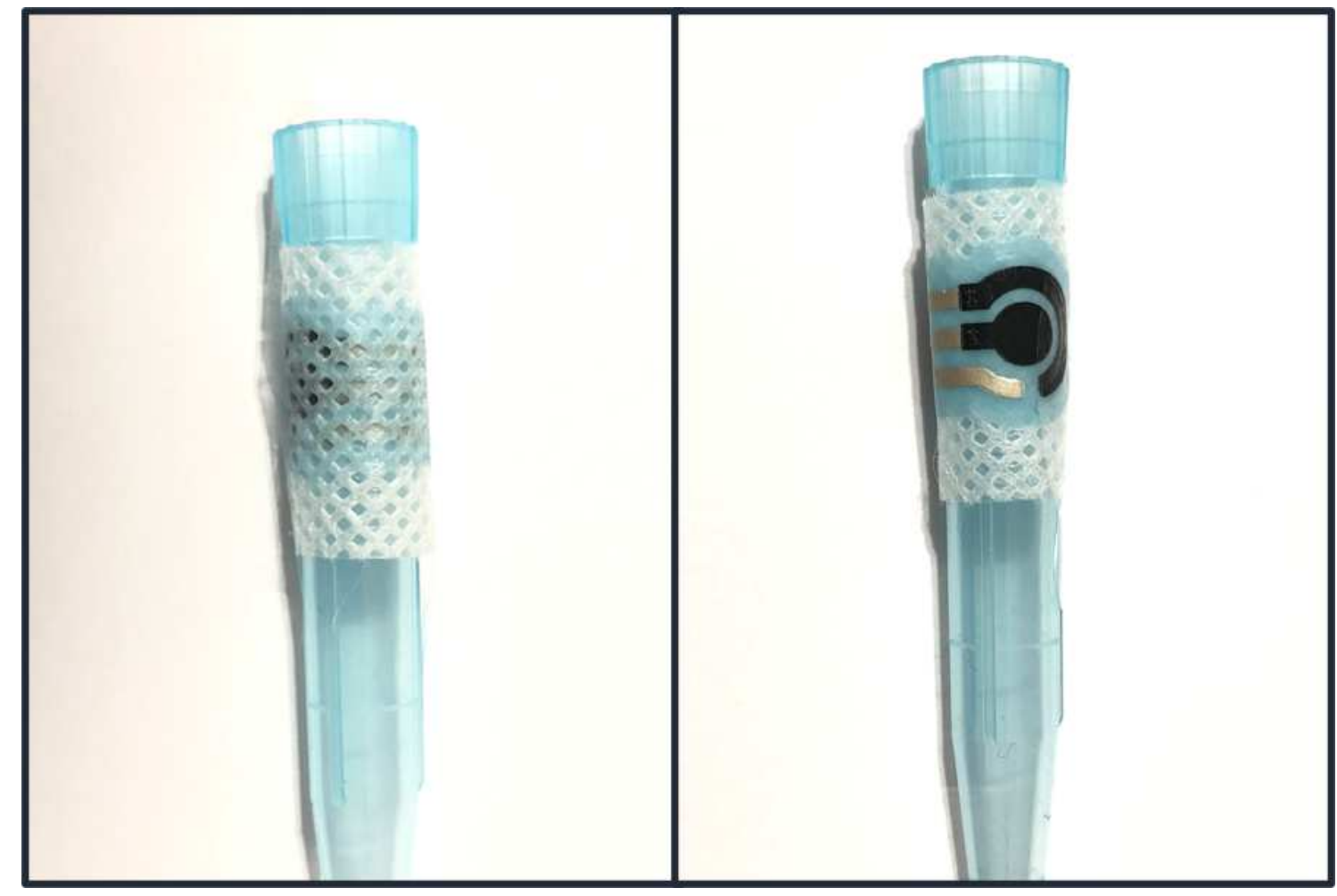

Figure 6. The flexibility of the SPE design (C) printed on textiles
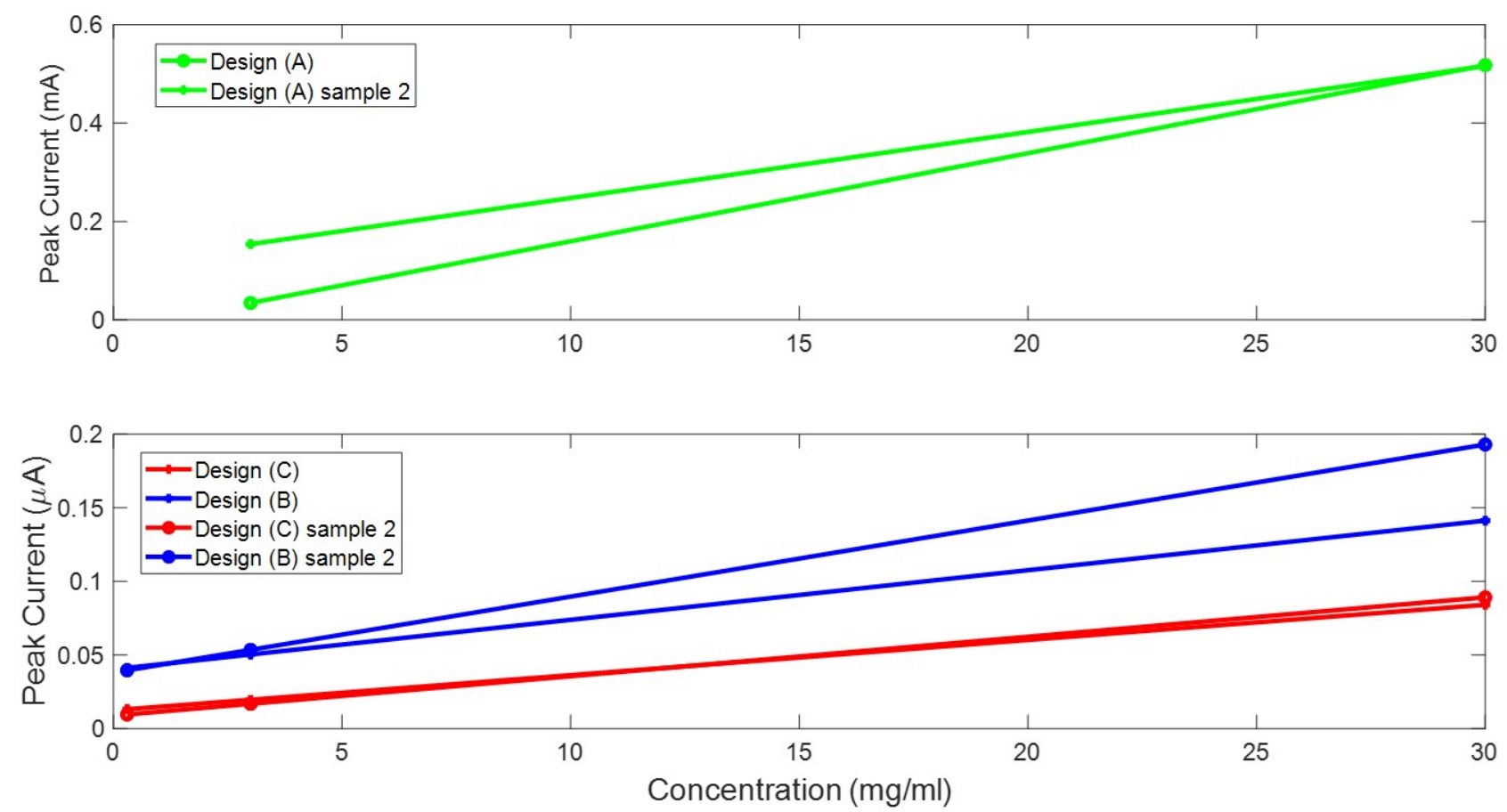

Figure 7. The oxidation peak observed after repeating the experiment twice using the three screen printed sensor designs

\section{Conclusion}

The sensor was fabricated using the screen printing technique and consisted of four layers: an interface layer, silver electrodes, carbon conducting interface area and an encapsulation layer. The sensor relied on quantifying the protein concentration by 


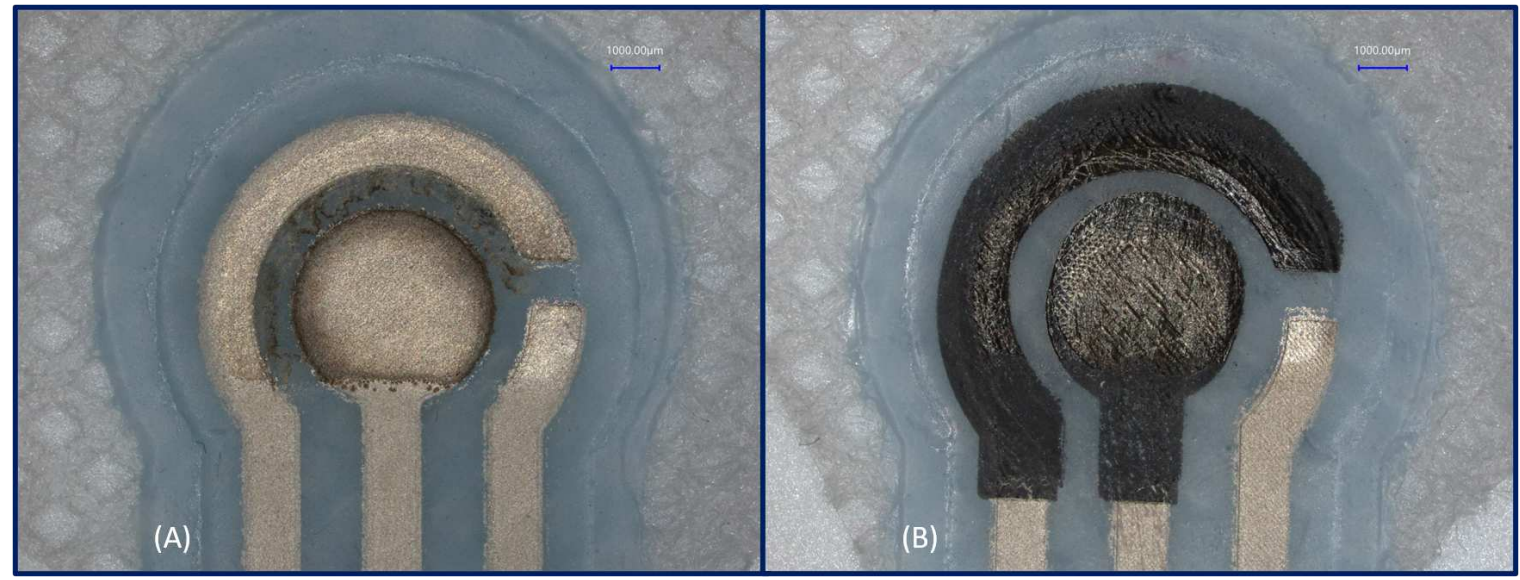

Figure 8. The silver containing SPEs after use (A) silver only based SPE and (B) Silver and Carbon SPE

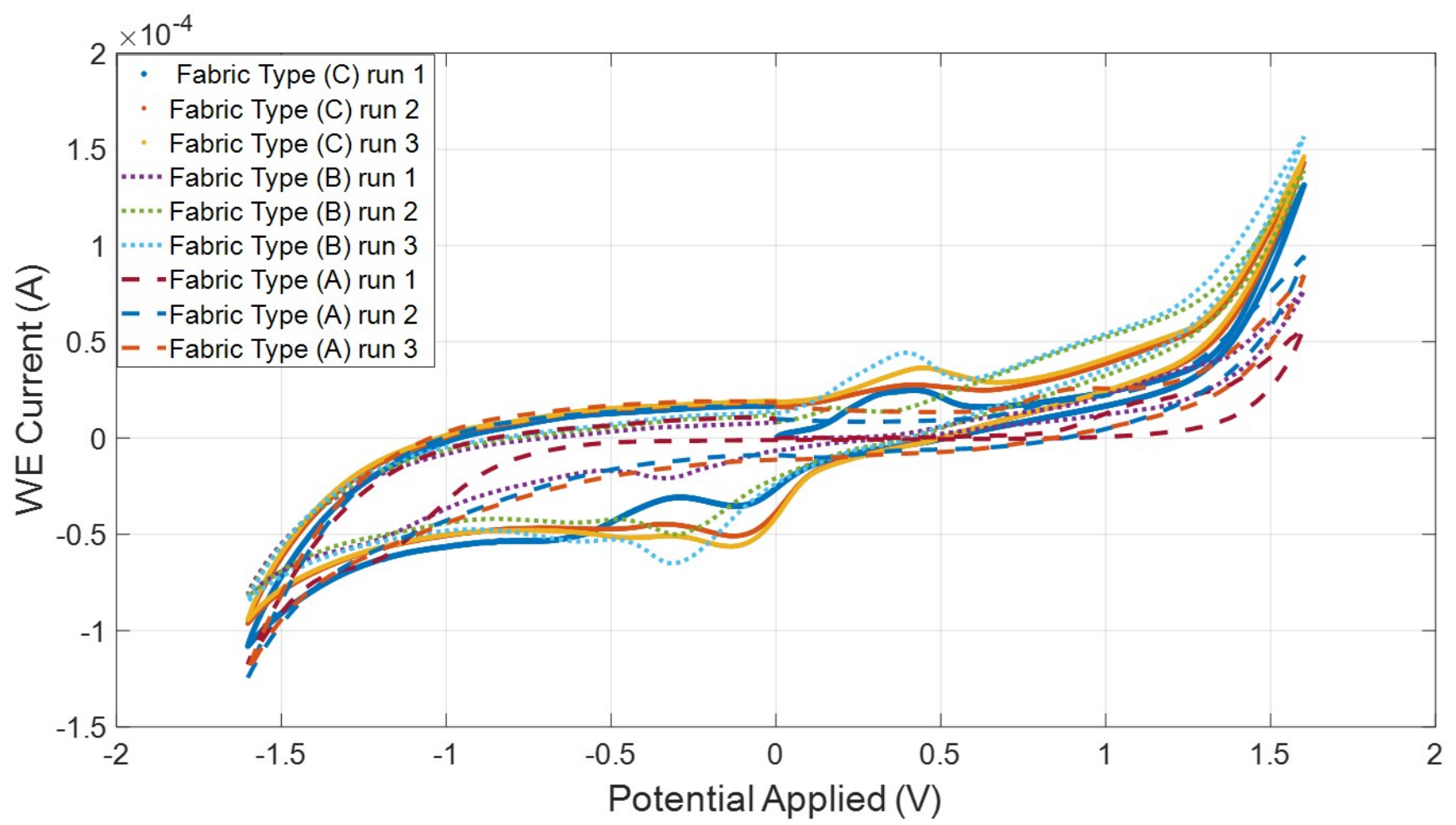

Figure 9. Measurements obtained when testing a sample of $30 \mathrm{mg} / \mathrm{ml}$ of BSA solutions on the third SPE design and conducting three cycles on the three types of fabrics

measuring changes to conducting cyclic voltammetry measurements. After testing three sensor designs with different silver and carbon compositions, it was concluded that that the SPE with only carbon material presented at the interface zone was the optimal design as not only did it provide the most reproducible and consistent results upon testing, it also remained unaffected by oxidation. The sensor was printed on three different types of fabric and all presented promising results, one of which was wound dressing fabric which was chosen as the substrate as it is commonly used medically. Finally, upon further cyclic voltammetry empirical testing and result analysis conducted using carbon only sensors (design (C)) and on Type C fabric, it was determined that to compare the outcome of different BSA concentrations it is best to take the average of the second and third cycles conducted on the same sensor. The cyclic voltammetry measurements demonstrated that the screen printed protein sensor could accurately monitor BSA concentrations from 0.3 to $30 \mathrm{mg} / \mathrm{ml}$ with a sensitivity of $0.0026 \mu \mathrm{A} / \mathrm{M}$. 


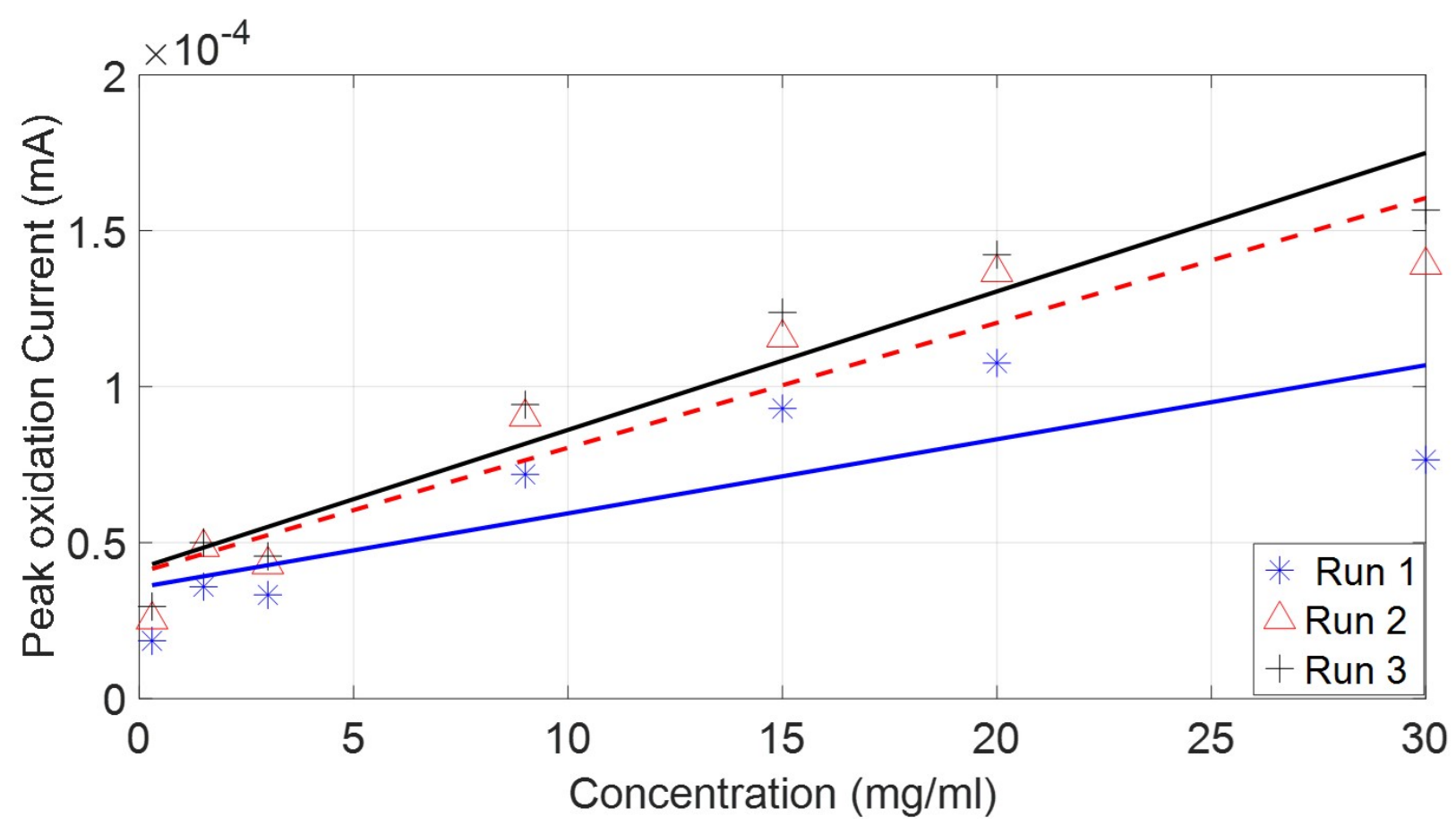

Figure 10. Measurements obtained when testing 8 BSA concentrations on the third SPE design and conducting 3 cycles for each sample on each sensor

\section{Methods}

\section{Screen printing process \\ Layer types}

To design the flexible protein sensors shown in Figure 6, the sensor consisted of four printed layers. Initially, an interface layer was built to create a base for the electrode conductive tracks. Silver electrodes were then deposited over the interface layer. In two of the designs (design B and C), carbon conductive ink was deposited over the interface zone. Finally, an encapsulation layer was placed on top of the sensor tracks and around the interface zone to protect the sensor electrodes and prevent any short circuit. In this work, three different designs were considered when designing the second conductive layer.

\section{Ink Types}

Three types of inks were used: a UV curable polymer ink from ElectraPolymers Ltd was used to act as the interface and encapsulation, carbon and silver inks from Henkel were used as the electrodes and conductive tracks, respectively.

\section{Textile materials}

Three different textile types were tested: 1) a non-woven polypropylene fabric (Type A), 2) a woven polyester/cotton fabric $(65 \% / 35 \%)$ (Type B), 3) a non-woven polyester fabric (Type C). Type A and C are commonly used in used in wound dressings and therefore are more favorable. Although the woven polyester/cotton (Type B) fabric is the most ideal type of fabric for printing because it is relatively smoother than the others (has areal surface roughness $(\mathrm{Sa})=59.37 \mu \mathrm{m}$ ), yet it is not commonly used in wound dressings.

\section{Protein solution preparation}

The protein used to test the fabricated sensors was BSA powder as it is considered as a standard for protein quantification (Sigma-Aldrich, Steinheim, Germany). In this research, 8 samples of BSA solutions were prepared with different concentrations and diluted using deionized water: $30,23,18,11,7,3,1,0.3 \mathrm{mg} / \mathrm{ml}$. Initially, $30 \mathrm{mg} / \mathrm{ml}$ was prepared by putting 3 grams of BSA powder in $100 \mathrm{~mL}$ of deionized water, and then the rest of the solutions were prepared by diluting the original stock solution.

\section{Measurement setup}

A setup similar to the methodology discussed in the previous work was implemented to evaluate the sensitivity shown in Table 1 of the developed textile-based screen printed carbon electrodes as shown in Figure 11. The printed protein sensor was connected 
the Metrohm Dropsens device. Ice glass containers were placed within a close proximity to the sensors to provide humid environment and to prevent evaporation of the BSA samples. This was achieved by conducting a series of cyclic voltammetry experiments on different protein solutions using an AUTOLAB potentiostat device (PGSTAT101). The setup parameters are listed in Table 2.

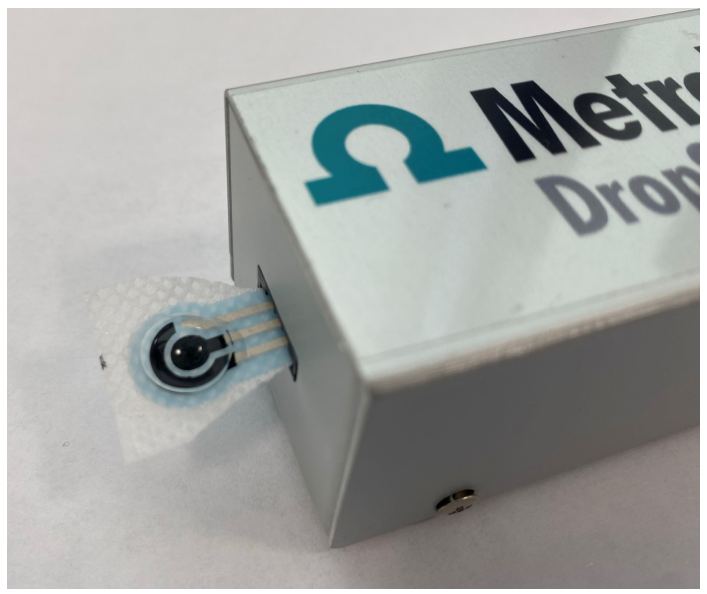

Figure 11. Using of textile screen printed electrode to measure protein concentration in BSA

\begin{tabular}{|c|c|}
\hline Parameter & Value \\
\hline Measurement type & CV staircase \\
\hline Scan rate & $0.08 \mathrm{~V} / \mathrm{s}$ \\
\hline Start potential & $0.0 \mathrm{~V}$ \\
\hline Step & $0.0025 \mathrm{~V}$ \\
\hline Upper vertex potential & $1.6 \mathrm{~V}$ \\
\hline Lower vertex potential & $-1.6 \mathrm{~V}$ \\
\hline
\end{tabular}

Table 2. Cyclic voltammetry measurement software setup parameters

\section{References}

1. Pourhanifeh, M. H., Mahdavinia, M., Reiter, R. J. \& Asemi, Z. Potential use of melatonin in skin cancer treatment: A review of current biological evidence. J. Cell. Physiol. 234, 12142-12148, DOI: https://doi.org/10.1002/jcp.28129 (2019).

2. Tang, N. et al. Wearable sensors and systems for wound healing-related ph and temperature detection. Micromachines 12, DOI: 10.3390/mi12040430 (2021).

3. Olsson, M. et al. The humanistic and economic burden of chronic wounds: A systematic review. Wound Repair Regen. 27, 114-125, DOI: 10.1111/wrr.12683 (2019).

4. Guest, J. F., Fuller, G. W. \& Vowden, P. Cohort study evaluating the burden of wounds to the uk's national health service in 2017/2018: update from 2012/2013. BMJ Open 10, DOI: 10.1136/bmjopen-2020-045253 (2020). https: //bmjopen.bmj.com/content/10/12/e045253.full.pdf.

5. Guest, J. F. et al. Health economic burden that different wound types impose on the uk's national health service. Int. Wound J. 14, 322-330, DOI: https://doi.org/10.1111/iwj.12603 (2017). https://onlinelibrary.wiley.com/doi/pdf/10.1111/iwj.12603.

6. Frykberg, R. G. \& Banks, J. Challenges in the treatment of chronic wounds. Adv. Wound Care 4, 560-582, DOI: 10.1089/wound.2015.0635 (2015). PMID: 26339534, https://doi.org/10.1089/wound.2015.0635.

7. Pal, A. et al. Early detection and monitoring of chronic wounds using low-cost, omniphobic paper-based smart bandages. Biosens. Bioelectron. 117, 696-705, DOI: https://doi.org/10.1016/j.bios.2018.06.060 (2018).

8. Sen, C. K. et al. Human skin wounds: A major and snowballing threat to public health and the economy. Wound Repair Regen. 17, 763-771, DOI: https://doi.org/10.1111/j.1524-475X.2009.00543.x (2009). https://onlinelibrary.wiley.com/doi/ pdf/10.1111/j.1524-475X.2009.00543.x. 
9. Farooqui, M. \& Shamim, A. Low cost inkjet printed smart bandage for wireless monitoring of chronic wounds. Sci. Reports 6, 28949, DOI: 10.1038/srep28949 (2016).

10. Gao, Y., Yu, L., Yeo, J. C. \& Lim, C. T. Flexible hybrid sensors for health monitoring: Materials and mechanisms to render wearability. Adv. Mater. 32, 1902133, DOI: https://doi.org/10.1002/adma.201902133 (2020). https://onlinelibrary.wiley. com/doi/pdf/10.1002/adma.201902133.

11. Hasan, M. M. \& Hossain, M. Nanomaterials-patterned flexible electrodes for wearable health monitoring: a review. $J$. Mater. Sci. 56, DOI: 10.1007/s10853-021-06248-8 (2021).

12. Kan, C.-W. \& Lam, Y.-L. Future trend in wearable electronics in the textile industry. Appl. Sci. 11, DOI: 10.3390/ app11093914 (2021).

13. Zhao, J. et al. A naturally integrated smart textile for wearable electronics applications. Adv. Mater. Technol. 5, 1900781, DOI: https://doi.org/10.1002/admt.201900781 (2020).

14. Gupta, B., Agarwal, R. \& Alam, M. Textile-based smart wound dressings. Indian J. Fibre Textile Res. 35, 174-187 (2010).

15. Strehlitz, B., Nikolaus, N. \& Stoltenburg, R. Protein detection with aptamer biosensors. Sensors 8, 4296-4307, DOI: $10.3390 / \mathrm{s} 8074296$ (2008).

16. Zelada-Guillén, G. A. et al. Ultrasensitive and real-time detection of proteins in blood using a potentiometric carbonnanotube aptasensor. Biosens. Bioelectron. 41, 366-371, DOI: 10.1016/j.bios.2012.08.055 (2013).

17. Bocian, A. et al. Comparison of methods for measuring protein concentration in venom samples. Animals 10 (2020).

18. Hirst, N. A. Development of biosensors for early detection of anastomotic leak and sepsis (2014).

19. Karimi-Maleh, H. et al. A critical review on the use of potentiometric based biosensors for biomarkers detection. Biosens. Bioelectron. 184, 113252, DOI: https://doi.org/10.1016/j.bios.2021.113252 (2021).

20. Parsajoo, C., Kauffmann, J.-M. \& Elkaoutit, M. 9 - biosensors for drug testing and discovery. In Higson, S. (ed.) Biosensors for Medical Applications, Woodhead Publishing Series in Biomaterials, 233-262, DOI: https://doi.org/10.1533/ 9780857097187.2.233 (Woodhead Publishing, 2012).

21. Gerard, M., Chaubey, A. \& Malhotra, B. Application of conducting polymers to biosensors. Biosens. Bioelectron. 17, 345-359, DOI: https://doi.org/10.1016/S0956-5663(01)00312-8 (2002).

22. Seki, A., ichi Ikeda, S., Kubo, I. \& Karube, I. Biosensors based on light-addressable potentiometric sensors for urea, penicillin and glucose. Anal. Chimica Acta 373, 9-13, DOI: https://doi.org/10.1016/S0003-2670(98)00364-X (1998).

23. Jia, Y.-F. et al. Unlabeled multi tumor marker detection system based on bioinitiated light addressable potentiometric sensor. Analyst 137, 3806-3813, DOI: 10.1039/C2AN35188A (2012).

24. Poghossian, A. et al. Towards addressability of light-addressable potentiometric sensors: Shunting effect of non-illuminated region and cross-talk. Sensors Actuators B: Chem. 244, 1071-1079, DOI: https://doi.org/10.1016/j.snb.2017.01.047 (2017).

25. Akl, M. A., El-gharkawy, E.-S. R., El-mahdy, N. A., El-Sheikh, S. M. \& Sheta, S. M. A novel nano copper complex: potentiometry, $\mathrm{dft}$ and application as a cancer prostatic biomarker for the ultrasensitive detection of human psa. Dalton Trans. 49, 15769-15778, DOI: 10.1039/D0DT03318A (2020).

26. Ding, J. \& Qin, W. Recent advances in potentiometric biosensors. TrAC Trends Anal. Chem. 124, 115803, DOI: https://doi.org/10.1016/j.trac.2019.115803 (2020).

27. Coppedè, N. et al. Ion selective textile organic electrochemical transistor for wearable sweat monitoring. Org. Electron. 78, 105579, DOI: https://doi.org/10.1016/j.orgel.2019.105579 (2020).

28. Emaminejad, S. et al. Autonomous sweat extraction and analysis applied to cystic fibrosis and glucose monitoring using a fully integrated wearable platform. Proc. Natl. Acad. Sci. 114, 4625-4630, DOI: 10.1073/pnas.1701740114 (2017). https://www.pnas.org/content/114/18/4625.full.pdf.

29. Chung, H.-J. et al. Stretchable, multiplexed ph sensors with demonstrations on rabbit and human hearts undergoing ischemia. Adv. Healthc. Mater. 3, 59-68, DOI: https://doi.org/10.1002/adhm.201300124 (2014). https://onlinelibrary. wiley.com/doi/pdf/10.1002/adhm.201300124.

30. Bariya, M. et al. Roll-to-roll gravure printed electrochemical sensors for wearable and medical devices. ACS Nano 12, 6978-6987, DOI: 10.1021/acsnano.8b02505 (2018). PMID: 29924589, https://doi.org/10.1021/acsnano.8b02505.

31. Choi, D.-H. et al. Sweat test for cystic fibrosis: Wearable sweat sensor vs. standard laboratory test. J. Cyst. Fibros. 17, e35-e38, DOI: https://doi.org/10.1016/j.jcf.2018.03.005 (2018). 
32. Parrilla, M. et al. Wearable all-solid-state potentiometric microneedle patch for intradermal potassium detection. Anal. Chem. 91, 1578-1586, DOI: 10.1021/acs.analchem.8b04877 (2019). https://doi.org/10.1021/acs.analchem.8b04877.

33. Ibanez Labiano, I. et al. Screen Printing Carbon Nanotubes Textiles Antennas for Smart Wearables. Sensors 21, 4934, DOI: $10.3390 / \mathrm{s} 21144934(2021)$.

34. Musa, A. M., Kiely, J., Luxton, R. \& Honeychurch, K. C. Recent progress in screen-printed electrochemical sensors and biosensors for the detection of estrogens. TrAC Trends Anal. Chem. 139, 116254, DOI: https://doi.org/10.1016/j.trac.2021. 116254 (2021).

35. Matzeu, G. et al. An integrated sensing and wireless communications platform for sensing sodium in sweat. Anal. Methods 8, 64-71, DOI: 10.1039/C5AY02254A (2016).

36. McCaul, M. et al. Wearable platform for real-time monitoring of sodium in sweat. ChemPhysChem 19, 1531-1536, DOI: https://doi.org/10.1002/cphc.201701312 (2018).

37. Mohamed, H. M. Screen-printed disposable electrodes: Pharmaceutical applications and recent developments. TrAC Trends Anal. Chem. 82, 1-11, DOI: https://doi.org/10.1016/j.trac.2016.02.010 (2016).

38. Salvo, P. et al. Sensors and biosensors for c-reactive protein, temperature and ph, and their applications for monitoring wound healing: A review. Sensors 17 (2017).

39. Guinovart, T., Valdés-Ramírez, G., Windmiller, J. R., Andrade, F. J. \& Wang, J. Bandage-based wearable potentiometric sensor for monitoring wound ph. Electroanalysis 26, 1345-1353, DOI: https://doi.org/10.1002/elan.201300558 (2014).

40. James, T. J., Hughes, M. A., Cherry, G. W. \& Taylor, R. P. Simple biochemical markers to assess chronic wounds. Wound Repair Regen. 8, 264-269, DOI: https://doi.org/10.1046/j.1524-475x.2000.00264.x (2000).

41. ElSaboni, Y., Hunt, J. A., Moffatt, C. \& Wei, Y. Empirical model for identifying protein concentrations in wound using cyclic voltammetry. IEEE Sensors Lett. 5, 1-4, DOI: 10.1109/LSENS.2021.3119420 (2021).

42. Mukhin, N. et al. Label-free protein detection by micro-acoustic biosensor coupled with electrical field sorting. theoretical study in urine models. Sensors 21, DOI: 10.3390/s21072555 (2021).

43. Caraceni, P., Tufoni, M. \& Bonavita, M. Clinical use of albumin. Blood transfusion = Trasfusione del sangue 11 Suppl 4 , s18-25, DOI: 10.2450/2013.005s (2013).

44. Levitt, D. \& Levitt, M. Human serum albumin homeostasis: A new look at the roles of synthesis, catabolism, renal and gastrointestinal excretion, and the clinical value of serum albumin measurements. Int. J. Gen. Medicine Volume 9, 229-255, DOI: 10.2147/IJGM.S102819 (2016).

45. Edsberg, L. E., Wyffels, J. T., Ogrin, R., Craven, B. C. \& Houghton, P. A pilot study evaluating protein abundance in pressure ulcer fluid from people with and without spinal cord injury. The J. Spinal Cord Medicine 38, 456-467 (2015).

46. Legendre, C. et al. Impact of protein deficiency on venous ulcer healing. J. Vasc. Surg. 48, 688-693, DOI: https: //doi.org/10.1016/j.jvs.2008.04.012 (2008).

47. Lee, H. J. \& Cheng, J.-X. 5 - label-free stimulated raman scattering imaging of neuronal membrane potential. In Alfano, R. R. \& Shi, L. (eds.) Neurophotonics and Biomedical Spectroscopy, Nanophotonics, 107-122, DOI: https: //doi.org/10.1016/B978-0-323-48067-3.00005-6 (Elsevier, 2019).

48. Zhang, D. et al. Protein detecting with smartphone-controlled electrochemical impedance spectroscopy for point-of-care applications. Sensors Actuators B: Chem. 222, 994-1002, DOI: https://doi.org/10.1016/j.snb.2015.09.041 (2016).

49. Nomura, K.-i., Horii, Y., Kanazawa, S., Kusaka, Y. \& Ushijima, H. Fabrication of a textile-based wearable blood leakage sensor using screen-offset printing. Sensors 18, DOI: 10.3390/s18010240 (2018). 